NOTE

\title{
(Vapour + liquid) equilibria of (fluoromethane + tetrafluoromethane) at the temperature $130.46 \mathrm{~K}$
}

\author{
I. M. A. Fonseca, G. G. Sardinha, and L. Q. Lobo \\ Departamento de Engenharia Química, Universidade de Coimbra, \\ 3000 Coimbra, Portugal
}

\begin{abstract}
The total vapour pressure of binary liquid mixtures of (fluoromethane + tetrafluoromethane) has been measured at the temperature $130.46 \mathrm{~K}$. Two partially miscible liquid phases were found. The excess molar G ibbs energy was calculated in the region where the two liquids are mutually soluble. For the hypothetical equimolar mixture, $G_{\mathrm{m}}^{\mathrm{E}}(x=0.5)=846 \mathrm{~J} \cdot \mathrm{mol}^{-1}$. () 1998 A cademic Press
\end{abstract}

KEY WORDS: fluorocarbons; vapour pressure; (liquid + liquid) immiscibility; low temperature

\section{Introduction}

In recent papers ${ }^{(1-3)}$ we have published experimental VLE data for $\left\{x \mathrm{CH}_{3} \mathrm{~F}+\right.$ $(1-x) \mathrm{Xe}\}$ and $\left\{x \mathrm{CH}_{3} \mathrm{~F}+(1-x) \mathrm{N}_{2} \mathrm{O}\right\}$. While the former is a (dipolar + nonpolar) mixture (since fluoromethane has an almost pure dipolar molecule: dipole moment $\mu=6.2 \cdot 10^{-30} \mathrm{C} \cdot \mathrm{m}$; quadrupole moment $Q=0.13 \cdot 10^{-40} \mathrm{C} \cdot \mathrm{m}^{2}$ ), the latter may be considered of the type (dipolar + quadrupolar) (bearing in mind that $\mathrm{N}_{2} \mathrm{O}$ molecules have a small dipole moment: $\mu=0.55 \cdot 10^{-30} \mathrm{C} \cdot \mathrm{m} ; \quad Q=$ $\left.12.3 \cdot 10^{-40} \mathrm{C} \cdot \mathrm{m}^{2}\right)$. These measurements suggested the study of the system $\left\{x \mathrm{CH}_{3} \mathrm{~F}\right.$ $\left.+(1-x) \mathrm{CF}_{4}\right\}$ which may be considered a mixture of the (dipolar + octopolar) type $\left(\mathrm{CF}_{4}\right.$ : octopole moment $\left.\Omega=16.0 \cdot 10^{-50} \mathrm{C} \cdot \mathrm{m}^{3}\right){ }^{(4)} \mathrm{Croll}$ and $\mathrm{Scott}\left({ }^{(5)}\right.$ measured the total vapour pressures for seven binary liquid mixtures containing fluorocarbon compounds and found that some of them showed (liquid + liquid) immiscibility. Schneider and coworkers ${ }^{(6)}$ also studied low-temperature systems involving $\mathrm{CF}_{4}$ and $\mathrm{CHF}_{3}$, but at much higher pressures (up to $p=150 \mathrm{M} \mathrm{Pa}$ ).

\section{Experimental and results}

Fluoromethane and tetrafluoromethane were both $M$ atheson products with a stated mole fraction purity of 0.990 . The two components were purified by 
TABLE 1. Vapour pressure $p$ and excess molar $\mathrm{Gibbs}$ energy $G_{\mathrm{m}}^{\mathrm{E}}$ for $\left\{x \mathrm{CH}_{3} \mathrm{~F}+(1-x) \mathrm{CF}_{4}\right\}$ at $T=130.46 \mathrm{~K} . x$ and $y$ are the mole fractions of methyl fluoride in the liquid and vapour phases, respectively, $z$ is the overall (liquid $\alpha$ + liquid $\beta$ +vapour) mole fraction of methyl fluoride in the system, $\delta p=p-p_{\text {calc }}$, where $p_{\text {calc }}$ is the calculated vapour pressure from equation (1), and $p$ is the measured equilibrium pressure

\begin{tabular}{lccccc}
\hline \multicolumn{1}{c}{$z$} & $x$ & $y$ & $p / \mathrm{kPa}$ & $\delta p / \mathrm{kPa}$ & $G_{\mathrm{m}}^{\mathrm{E}} /\left(\mathrm{J} \cdot \mathrm{mol}^{-1}\right)$ \\
\hline 0 & 0 & 0 & $32.341^{a}$ & 0 & 0 \\
0.0780 & & & 31.085 & & \\
0.1060 & & & 30.847 & & \\
0.1680 & & & 30.713 & & \\
0.2066 & & 30.689 & & \\
0.2597 & & 30.783 & & \\
0.3113 & & 30.726 & & \\
0.3909 & & 31.077 & & 195 \\
0.4443 & & & 30.796 & & \\
0.4975 & 0.9395 & 0.0098 & 30.822 & 0.718 & \\
& 0.9423 & 0.0097 & 30.834 & -0.736 & \\
1 & 1 & 1 & $0.381^{b}$ & 0 & \\
\hline
\end{tabular}

${ }^{a}$ To be compared with $p=32.228 \mathrm{kPa}$ from reference $8 .{ }^{b}$ The literature values are $p=(0.379 \pm$ $0.003) \mathrm{kPa}$ in reference 7 , and $p=(0.400 \pm 0.005) \mathrm{kPa}$ in reference 10.

fractionation in the laboratory low-temperature distillation column, with the middle fraction being used in the experiments. The final purity of the samples was confirmed by the constancy of the triple point pressure during melting in the usual way. ${ }^{(1,3)}$ The equilibrium pressures of the mixtures were measured at $T=130.46 \mathrm{~K}$, the triple-point temperature of fluoromethane. ${ }^{(7)}$ The apparatus and the technique used in the experiments have been described elsewhere. ${ }^{(1,3)}$ Two partially miscible liquid phases have been found. The experimental results are given in table 1 . In this table $z$ is the overall (liquid $\alpha+$ liquid $\beta$ +vapour) mole fraction of $\mathrm{CH}_{3} \mathrm{~F}$ in the system. The existence of two liquid layers is clearly shown by the constancy of the measured vapour pressures over almost the entire composition range, as shown in figure 1. In the limited region for which the two liquids are mutually soluble the excess molar $\mathrm{G}$ ibbs energy $G_{\mathrm{m}}^{\mathrm{E}}$ was calculated assuming that it could be represented by a one-parameter ( $A$ ) function of composition,

$$
G_{\mathrm{m}}^{\mathrm{E}}=R T A x(1-x)
$$

where $x$ is the mole fraction of $\mathrm{CH}_{3} \mathrm{~F}$ in the liquid phase. The value of $A$ found by minimizing the pressure residuals by Marquardt's method is $A=3.119$. The standard deviation of the pressure is $1.028 \mathrm{kPa}$. F or the (hypothetical) equimolar liquid mixture $G_{\mathrm{m}}^{\mathrm{E}}(x=0.5)=846 \mathrm{~J} \cdot \mathrm{mol}^{-1}$. The molar volumes of the pure liquids were taken from references 7 and 8; allowance was made for the imperfection of the two gases by considering the second virial coefficients given in the same references. 

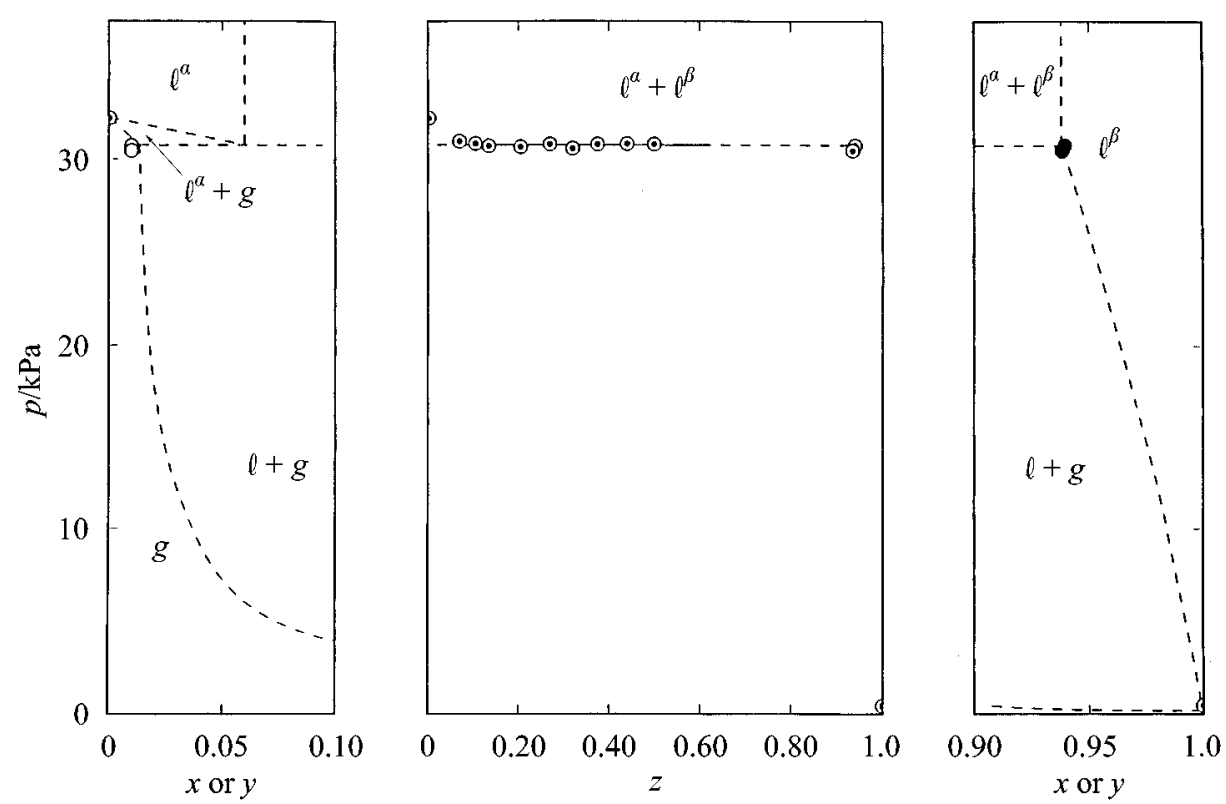

FIGURE 1. (V apour + liquid) equilibria of $\left\{x \mathrm{CH}_{3} \mathrm{~F}+(1-x) \mathrm{CF}_{4}\right\}$ at $T=130.46 \mathrm{~K} . x$ and $y$ are the calculated mole fractions of $\mathrm{CH}_{3} \mathrm{~F}$ in the liquid and vapour phases, respectively, $z$ is the overall $\left(\left.\right|^{\alpha}+\left.\right|^{\beta}+g\right)$ mole fraction of $\mathrm{CH}_{3} \mathrm{~F}$ in the system, and $p$ is the experimental vapour pressure. $\odot$, raw data; $\bullet$, liquid phase; $\bigcirc$, vapour phase; - . . - - , calculated phase boundary lines. $N$ ote the different scales along the mole fraction axis.

The system exhibits heterozeotropy. ${ }^{(9)}$ To calculate the mole fractions at the heterogeneous azeotrope we have considered the equilibrium between the two saturated liquid solutions, i.e.

$$
\begin{aligned}
\gamma_{1}^{\alpha} x^{\alpha} & =\gamma_{1}^{\beta} x^{\beta}, \\
\gamma_{2}^{\alpha}\left(1-x^{\alpha}\right) & =\gamma_{2}^{\beta}\left(1-x^{\beta}\right),
\end{aligned}
$$

where $\gamma_{i}$ is the activity coefficient of component $i\left(1\right.$ for $\mathrm{CH}_{3} \mathrm{~F}$, and 2 for $\left.\mathrm{CF}_{4}\right)$. The resulting equilibrium compositions are $x^{\alpha}=0.0606$ and $x^{\beta}=0.9384$, and the measured equilibrium pressure is $p=(30.838 \pm 0.295) \mathrm{kPa}$.

The composition of the vapour in equilibrium with the saturated liquid mixtures was obtained from the thermodynamic equilibrium conditions:

$$
\begin{gathered}
\varphi_{1} y p=\gamma_{1}^{\alpha} x^{\alpha} p_{1}^{*}=\left(\gamma_{1}^{\beta} x^{\beta} p_{1}^{*}\right), \\
\varphi_{2}(1-y) p=\gamma_{2}^{\alpha}\left(1-x^{\alpha}\right) p_{2}^{*}=\left\{\gamma_{2}^{\beta}\left(1-x^{\beta}\right) p_{2}^{*}\right\},
\end{gathered}
$$

where the asterisk refers to the pure substances. A two-term virial equation of state was used in the calculation of the fugacity coefficients $\varphi_{i}$. The composition of the vapour at the heterogeneous azeotrope is obtained as $y=(0.016 \pm 0.001)$. 


\section{REFERENCES}

1. Fonseca, I. M. A .; L obo, L. Q. Fluid Phase Equilib. 1989, 47, 249-263.

2. Fonseca, I. M. A .; L obo, L. Q. J. Chem. Thermodynamics 1994, 26, 647-650.

3. Fonseca, I. M. A .; Lobo, L. Q. Fluid Phase Equilib. 1995, 113, 127-138.

4. Gray, C. G.; Gubbins, K. E. Theory of Molecular Fluids, V ol. 1. Oxford U niversity Press: N ew Y ork. 1984.

5. Croll, I. M .; Scott, R. L. J. Phys. Chem. 1964, 68, 3853-3860.

6. Paas, R.; Schneider, G. M. J. Chem. Thermodynamics 1979, 11, 267-276.

7. Fonseca, I. M. A .; Lobo, L. Q. Rev. Port. Quim. 1989, 31, 118-121.

8. Lobo, L. Q.; Staveley, L. A. K. J. Chem. Eng. Data 1981, 26, 404-407.

9. Van N ess, H. C.; A bbott, M. M. Classical Thermodynamics of Nonelectrolyte Solutions. M cG raw-H ill: N ew Y ork. 1982, p. 396.

10. Fonseca, I. M. A.; Lobo, L. Q. J. Chem. Thermodynamics 1994, 26, 671-672.

(Received 3 February 1997; in final form 19 May 1998)

W A 97/011 